\title{
A PERCEPTUAL STUDY OF APPROXIMATED CANTONESE TONE CONTOURS
}

\author{
Yujia LI and Tan LEE \\ Department of Electronic Engineering, The Chinese University of Hong Kong, Hong Kong \\ yjli@ee.cuhk.edu.hk, tanlee@ee.cuhk.edu.hk
}

\begin{abstract}
This paper describes a perceptual study on approximated Cantonese tone contours. It is found that Cantonese tone contours and tone transitions can be approximated by a limited number of linear movements, without creating any noticeable perceptual difference. The slopes of these linear movements are analyzed. They are found to be related with two thresholds of pitch movement perception. The results of perceptual tests with polysyllabic words over large segmental variation confirm the feasibility of approximating F0 contours of Cantonese speech.
\end{abstract}

Index Terms - Tone perception, Cantonese tones, perceptual equivalence, speech naturalness

\section{INTRODUCTION}

Speech is transmitted in a path from human production to acoustics and from acoustics to human perception. The knowledge about production-acoustics relation has been largely explored in previous research, while that about perception with acoustics is relatively less understood. As production and perception are processed by different physical systems of human being [1], much of the knowledge should be very different when learning how to control acoustics by each individually. For example, in tone production, it was found that human articulators limit the maximum speed of pitch change [2]; in tone perception, the same tone contour can be perceived as a reversed target, if the context is different [3]. For speech applications aiming at perception, e.g., text-to-speech and speech enhancement, investigations on capturing perceptionsensitive acoustic features would be very helpful.

Tone has multiple functions in tonal languages. As a part of lexicon, they determine the meaning of a spoken word by aligning distinctive pitch patterns over a syllable. In real speech, tone is realized as an F0 contour, generally with great deviation from the phonological standard form, due to the influences from many factors. The realization of tones is said to be highly correlated with perceived naturalness of speech [4][5]. In non-tonal languages, tone-related perception research focuses on the development of intonation model [5][6]. In tonal languages, acoustic features for tone identification have been mostly investigated [7][8]. These features are language-dependent. Cantonese is well known for its richness in tone. Figure 1 gives the representative pitch patterns of the six tones in Cantonese [9]. The pitch patterns can be considered as the underlying targets for speech production to reach, and/or for speech perception to identify. "Entering tone" is carried by syllables ending with an occlusive coda $/ \mathrm{p} /, / \mathrm{t} /$ or $/ \mathrm{k} /$. They are contrastively shorter in duration but overlap with a non-entering counterpart in terms of pitch level. Most of Cantonese tones are level tones. Their discrimination greatly relies on the pitch heights in relation to each other.

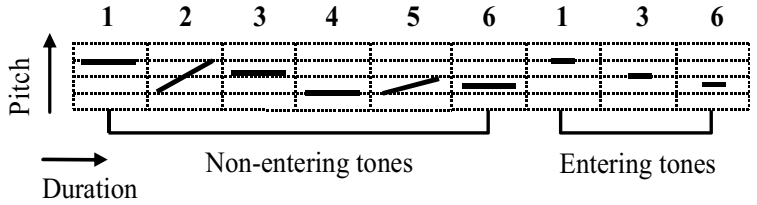

Figure 1: Pitch patterns of Cantonese tones.

Our research aims at discovering perception-sensitive acoustic properties from greatly varied tone contours, in terms of perceived speech naturalness. Considering the relation between production and perception, whether perception can differentiate every minute variation in human produced acoustics is the question being asked. Previous research suggests that perception of pitch movement has a limitation in resolution. The existence of glissando threshold and differential glissando threshold indicates that not all pitch movements can be perceived and not all of the perceived movements can be differentiated from each other [5]. We believe that the perception of tone contours relies mainly on the major trend of pitch movements, and is not sensitive to the exact F0 values at particular time instants. Our previous study [10] showed that Cantonese tone contours can be approximated by a few line segments, without creating noticeable perceptual difference. In this paper, we will analyze the approximated tone contours and relate the results of perceptual test with the glissando threshold. Furthermore, perceptual tests with a large variety of polysyllabic words are carried out to verify the general applicability of the approximation rules.

\section{ANALYSIS OF APPROXIMATED TONE CONTOURS}

\subsection{Perceptual equivalence of tone contours}

In our previous investigation, perceptual tests with linearly approximated tone contours were performed [10]. There were three different tests, which involved isolated syllables (Test 1), disyllabic words (Test 2), and long sentences (Test 3) respectively. For Test 1, three base syllables /wai/, /ji/, /jing/ ${ }^{1}$ were used. Each of them can be associated with six different tones, leading to 18 meaningful Chinese characters as shown in Table 1. For Tone 1, 3, 4 and 6, two types of approximation were tested: level (denoted by La) and falling (denoted by Fa). For Tone 2 and 5, three different approximations were formed, namely rising (Ra), level-rising (LRa) and falling-rising (FRa). Thus there are a total of 14 approximations for each base syllable. In Test 2, 44 disyllabic words with conflict tone boundary [3] were used. All syllables carry non-entering tones. In addition to the approximated tone contours for individual syllables, the inter-syllable transition was approximated also with a linear movement. In Test 3, the practicality of the approximations was evaluated with a few long

\footnotetext{
${ }^{1}$ The Cantonese syllables are transcribed with Jyut Ping (粵括) symbols
} 
sentences of natural Cantonese. In all of the tests, each modified utterance with approximated F0 contour was paired with the correspondent natural speech and presented to the subjects. The subjects were asked to select the better one or rate them as "same".

Table 1: List of 18 carrier syllables in Test 1 .

\begin{tabular}{|c|c|c|c|}
\hline \multirow{2}{*}{ Tone } & \multicolumn{3}{|c|}{ Base syllable } \\
\cline { 2 - 4 } & wai & ji & jing \\
\hline 1 & 威 & 醫 & 英 \\
\hline 2 & 委 & 椅 & 影 \\
\hline 3 & 畏 & 意 & 應 \\
\hline 4 & 維 & 怡 & 形 \\
\hline 5 & 偉 & 耳 & 郢 \\
\hline 6 & 惠 & 二 & 認 \\
\hline
\end{tabular}

\subsection{Analysis of approximated tone contours}

The results of the perceptual tests described above led to the following preliminary conclusions:

1. For competitive perception, level tones can be approximated by a single linear movement;

2. Two movements are necessary for rising tones;

3. One linear movement is adequate for inter-syllable transition.

The results of Test 1 are analyzed in more detail. Figure 2 gives the syllable-dependent test results. The preference votes to "natural", "modified" and "same" are illustrated by different colors and textures on the same vertical bar. The markers " 1 ", "2" and "3" refer to different base syllables. Generally, for Tone 1 and Tone 3, both La and Fa can reach equivalent perception; for Tone 4 and Tone 6, only $\mathbf{F a}$ is acceptable. As for the rising tones, $\mathbf{R a}$ is not appropriate and FRa gives the competitive perception. Except that Fa of Tone 6 of /wai/ got fewer votes on "same" and LRa and FRa of Tone 2 of /ji/ are equally preferred, no other significant difference is found among different base syllables. The above general conclusions are equally effective for the three syllables in our test.

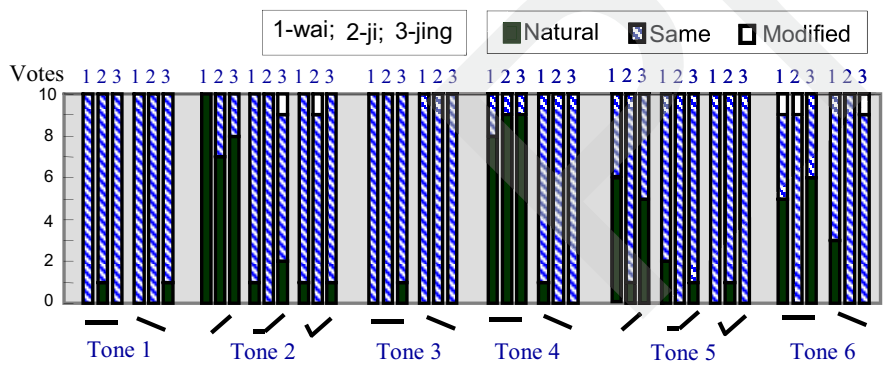

Figure 2: Syllable-dependent perceptual results of Test 1 .

A linear movement is characterized mainly by the slope and the height. How the slope of a pitch contour is correlated with perception has been studied since 1960s. Glissando threshold and differential glissando threshold are two major findings towards this question [5]. Glissando threshold is an absolute threshold of perceivable pitch change. If the slope of a pitch movement is below the threshold, no pitch change would be perceived.

The Glissando threshold is computed by

$$
G_{t}=0.16 / T^{2}
$$

where $G_{t}$ is in semitones per second, and $T$ is duration. The differential glissando threshold concerns the differentiation between two successive pitch movements. In [5], it was said that "In speech, two just distinguishable rates of change of FO will differ by a factor of at least two". The measurements of both thresholds were done with simple synthetic vowels or $\mathrm{CV}$ sequences.

Table 2 gives the value of measured slope in each of the approximations that we tested. Comparing Figure 2 with Table 2, the measured slopes are found to be correlated with the perceptual results. For Ra of Tone 2, Ra of Tone 5 and Fa of Tone 6, the inconsistent results among the syllables are related to the very different values of slopes

Table 2: Measured slopes of approximated tone contours.

\begin{tabular}{|c|c|c|c|c|c|c|c|c|c|}
\hline \multicolumn{10}{|c|}{ Slope $(\mathrm{Hz} / \mathbf{s})$} \\
\hline & Tone & \multicolumn{2}{|c|}{ Tone 1} & \multicolumn{4}{|c|}{ Tone 2} & \multicolumn{2}{|c|}{ Tone 3} \\
\hline \multicolumn{2}{|c|}{ Approximation } & La & $\mathbf{F a}$ & $\mathbf{R a}$ & LRa & FR & & La & $\mathbf{F a}$ \\
\hline \multirow{3}{*}{ 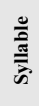 } & 1: wai & -0.95 & -17.21 & 300.09 & \begin{tabular}{l|l}
$-1 \#$ & $580.28 *$ \\
\end{tabular} & $-125.11 \#$ & $597 *$ & -0.08 & -43.90 \\
\hline & $2: \mathrm{ji}$ & -0.35 & -11.41 & 178.06 & \begin{tabular}{|c|c|c|}
$-2.09 \#$ & $296.8^{*}$
\end{tabular} & $-249 \#$ & $283.48^{*}$ & -3.58 & -52.78 \\
\hline & 3: jing & -3.57 & -74.41 & 205.59 & $-4.44 \# 338.26^{*}$ & -289.94\# & $365.48 *$ & -3.84 & -72.16 \\
\hline \multicolumn{10}{|c|}{ Slope $(\mathrm{Hz} / \mathbf{s})$} \\
\hline \multicolumn{2}{|r|}{ Tone } & \multicolumn{2}{|c|}{ Tone 4} & \multicolumn{4}{|c|}{ Tone 5} & \multicolumn{2}{|c|}{ Tone 6} \\
\hline \multicolumn{2}{|c|}{ Approximation } & $\mathrm{La}$ & $\mathbf{F a}$ & $\mathbf{R a}$ & LRa & FR & & La & $\mathbf{F a}$ \\
\hline \multirow{3}{*}{ 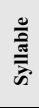 } & 1: wai & -0.58 & -120.06 & 140.94 & $-0.63 \# 211.6 *$ & -134.56\# & $189.05 *$ & 6.93 & -128.85 \\
\hline & $2: \mathrm{ji}$ & -2.33 & -165.97 & 58.26 & -2.09\# $103.48^{*}$ & -269.08\# & $98.04 *$ & -6.71 & -63.00 \\
\hline & 3: jing & -3.27 & -223.12 & 125.49 & $3.81 \#: 234.27 *$ & -177.19\# & $230.64 *$ & -12.95 & -53.02 \\
\hline
\end{tabular}

\# indicates the slope of the first movement in rising tones;

* indicates the slope of the second movement in rising tones.

The slope values are further compared with the glissando threshold and differential glissando threshold. In the tests, the average tone duration is $0.37 \mathrm{~s}$, leading to an estimated glissando threshold $1.17 \mathrm{ST} / \mathrm{s}$, or $14 \mathrm{~Hz} / \mathrm{s}$ (calculated by $D=12 \log _{2}\left(f / f_{r}\right)$, where $D$ is the distance between $f$ and $f_{r}$ in semitones, and $f_{r}$ is the reference frequency, which is defined as the height of Tone 3 , around $200 \mathrm{~Hz}$ for this particular speaker). As all the approximations of a tone are perceptually compared with the natural one, similar results would imply that these approximations are not perceptually distinguishable; otherwise the results should be different. The following observations are made:

1. If the slope of approximated contour is around the glissando threshold, e.g., Fa of Tone 1, the movement can not be perceived. Exceptionally, although the Fa slope of syllable /wai/ is much higher than the threshold, its movement cannot be perceived. Explanation can be associated with observation 2 .

2. Even when the slopes are $2-3$ times higher than the threshold, e.g., Tone 3 , the falling pitch movement can not be perceived. This indicates that complex speech may decrease the sensitivity of pitch perception such that the threshold is increased.

3. Fa of Tone 6 has similar value of slope with Tone 3, but these movements can be perceived. A possible explanation is that Tone 6 has a lower pitch level than Tone 3 and causes a lower threshold.

4. When the slopes are much higher than the glissando threshold, e.g., approximations for Tone 2, 5 and 4, the movements can be perceived. The ratios of pitch rising slopes between Tone 2 and Tone 5 , and those of pitch falling slopes between Tone 4 and Tone 6 are mostly between 2 to 3 , which is above the differential glissando threshold. Although the contours being compared were not presented in succession, they should be differentiable by perception, for the sake of speech recognition. For example, the similar slope values of $\mathbf{F a}$ of Tone 4 and Tone 6 for /wai/, map to worse perceptual results than the other two syllables, possibly because the two movements cannot be initially distinguished. 
In Test 2, totally 440 responses were collected, 10 for each word pair. $69.88 \%$ of the responses were for "same" and $13.36 \%$ for "modified", $16.75 \%$ for "natural". In $95.45 \%$ of the cases, modified utterances were considered to attain competitive perception, i.e., less than half of the votes were for "natural". The slopes of these approximated transitions for conflict tone boundaries are calculated and the results are shown as in Table 3. If one of the syllables carries a rising tone, the transitions are averagely less rapid. This may be due to that the post-transition rising tones start with a falling movement and thus reduce the conflict at tone boundaries. Transition slopes are found to have a large variance. Most of them are beyond the glissando threshold and expected to be perceivable. However, we believe that if transitions are caused by physiological constraints of speech production to generate a smooth pitch contour [2], perception would not be sensitive to these movements unless the slopes are higher than a certain value and so that the movement would be perceived as unnatural "jump" [5]. The highest transition slope in this test is beyond $815 \mathrm{~Hz} / \mathrm{s}$, which occurs both in unvoiced and voiced transitions. They have no side-effect to perception, suggesting the maximum transition slope, perception can afford to perceive speech from natural to unnatural, could be higher than this value.

Table 3: Statistics of the transition slopes $(\mathrm{Hz} / \mathrm{s})$ in Test 2.

\begin{tabular}{|c|c|c|c|c|}
\hline \multicolumn{5}{|c|}{ Total average slope: $367.59 ; \quad$ Standard deviation: 197.97} \\
\hline & Average & Std. & Max. & Min. \\
\hline Level tones only & 432.92 & 165.25 & 816.28 & 130.85 \\
\hline Rising tones included & 330.26 & 205.30 & 817.21 & 2.44 \\
\hline
\end{tabular}

\section{PERCEPTION OF POLYSYLLABIC WORDS WITH APPROXIMATED TONE CONTOURS}

In the above study, the test materials had very limited lexical and segmental variation. To further confirm the validity of our preliminary conclusions, a perceptual test with a large number of different polysyllabic words is carried out. We also aim at deriving the statistical properties of perceptually equivalent approximations and accordingly developing methods of automatically establishing the approximation.

We select 335 words from the CUWORD corpus [11]. These words are 4 to 6 syllables long and contain only level tones. The utterances were recorded by a female speaker. The natural F0 contour of each utterance was manually approximated following the rules found in the preliminary study. The modified speech with approximated F0 contour was generated by the software Praat [12]. To compensate the quality loss in synthesized speech, the F0 of both natural and synthesized speech were down-shifted by $10 \mathrm{~Hz}$. The testing procedures are similar with the preliminary study. About $10 \%$ "control" stimuli (very different stimuli in a pair and identical stimuli in a pair) were inserted into the list of test items. 26 subjects participated in the test. All of them are university students and native Cantonese speaker with normal hearing. Each subject went through half of the full list, plus additional control pairs, totally around 190 pairs. Each test lasted for about 40 minutes, including two mandatory 5-minute breaks. The results of six subjects were judged and discarded. The total number of effective responses was 3350, 10 for each word pair.

In the preference distribution, about $85 \%$ of the cases, either "modified" was preferred or the two stimuli were perceived as "same". The results for control stimuli show that some of the subjects tend to make random selection between "natural" and "modified", when they could not tell any difference between them.
If this is considered, the true distribution would have a higher percentage of "same" and lower percentage of both "natural" and "modified".

Figure 3 shows the distribution of the test words against different preference choices. For $94.53 \%$ of the words, the utterances with approximated F0 contours can attain competitive perception. Only $2.69 \%$ were found to be less preferred than the natural speech. No modified word is perceived better than the natural one, i.e., for all the words, their votes to "modified" were less than the half.
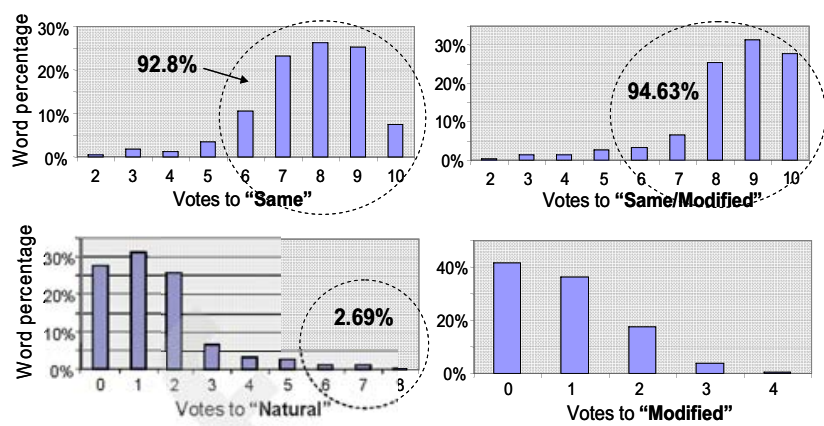

Figure 3: Word distribution over different preferences.

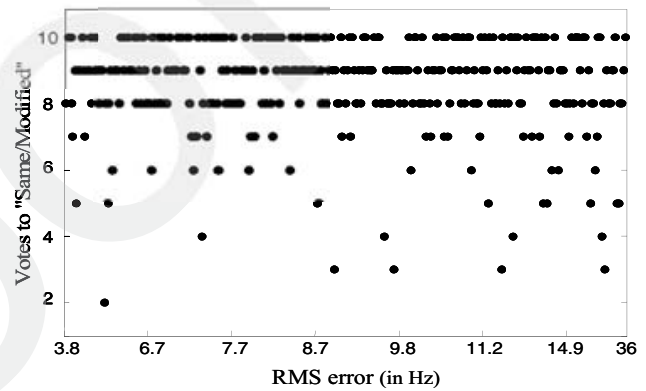

Figure 4: Comparison of the RMS error and perceptual preference.

The mean RMS error of approximated tones from the natural ones is $9.92 \mathrm{~Hz}$ and the standard deviation is $4.05 \mathrm{~Hz}$. Figure 4 relates the RMS error with perceptual test results. It seems that the RMS error is not closely correlated with perception. When the error increases, perceptual results exhibit irregular variation. In particular, the approximation with an RMS error of $36 \mathrm{~Hz}$ got 10 votes to "same", while the one having a small RMS error of $6 \mathrm{~Hz}$ got 8 votes to "natural". Nevertheless, the RMS error is useful in our study. An approximation, with small RMS error but unfavorable perception, is expected to contain some perceptionsensitive modifications; on the other hand, an approximation with large RMS error but equivalent perception would reveal perception-insensitive features.

Figure 5 gives the statistics of the slope values in approximated contours, for different tones and tone transitions. As expected, most slopes of tones show negative values (falling movement). The slopes of Tone 1 appear to be much smaller than others, average around level $(-4.5 \mathrm{~Hz} / \mathrm{s})$, also with more cases of rising (positive slopes). Slopes of Tone 4 are large, more than two times of those for Tone 3 and 6, also with a large dynamic range. Slopes of Tone 3 and 6 are similar. Pitch height rather than slope could be more critical in perceptual differentiation of them. Slopes of entering tones vary a lot and are generally much larger than that of non-entering counterpart. For the ease of comparison, slopes of transitions are all converted into negative absolute values. Both the cases of "between non-entering tones" and "preceding entering tones" have an average slope about $300 \mathrm{~Hz} / \mathrm{s}$, comparable with the 
finding in preliminary study. Unexpectedly, the case of "following entering tones" has a lower average slope of $197 \mathrm{~Hz} / \mathrm{s}$. High slope was initially supposed to appear as stop coda would introduce in an unvoiced transition after it and a F0 reset of the succeeding tone.

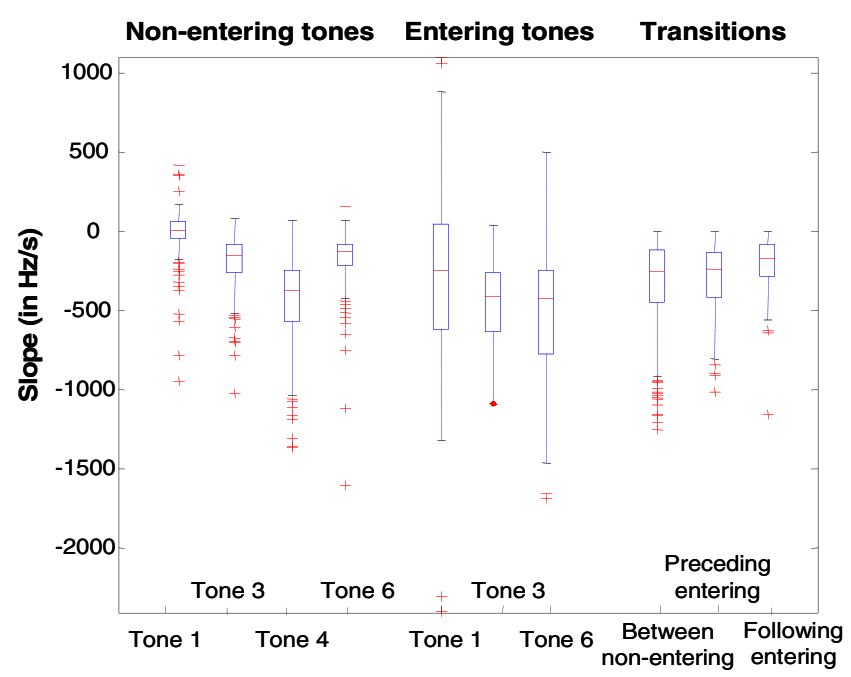

Figure 5: Statistics of slopes in approximations.

\section{DISCUSSION AND CONCLUSION}

The results of our perceptual tests show that Cantonese speech perception is not sensitive to the exact acoustic F0 values at individual time instants, but the major trend of pitch movement mostly affects it. In terms of competitive perception, these major trends can be approximated by a limited number of line segments, i.e., one movement for level tones, two movements for rising tones and one movement for tone transition. The results with a variety of polysyllabic words confirm the possibility of making linear approximations in natural speech from different speakers. As a major feature of linear movements, the slopes are analyzed. They are found to be closely correlated with perceived naturalness of speech, based on the theory of glissando threshold and differential glissando threshold.

Linear movements could potentially be a languageindependent representation of perception-sensitive properties. Except for the approximations we found for Cantonese, similar findings were ever reported in a perceptual study of non-tonal language [5]. In this study, perceptually equivalent "close copy" of natural intonation contour was manually determined by subjects. The "close copy" consists of syllable-sized line segments. Such simple approximations are desirable in both the fundamental study of prosody and the applications of prosody modeling. Linear movements lead the study of perception-sensitive properties to the limited features which determine the movements, the height, the slope, and the timing (locations of turning points in continuous speech). The simple contours will also largely decrease the cost of signal processing in $\mathrm{F} 0$ generation.

In [2], it is discussed that production probably generates unavoidably unnecessary acoustics. Hence, justified comparison is expected, in order to facilitate the investigation if the linear movements are more desirable by perception. However, in the current study, the quality loss in re-synthesized speech still cannot be totally balanced, which impedes a faired comparison between the natural and approximated contours. Possible solution is using TTS generated speech to carry both the contours, as stimuli in perceptual tests. Rising tones are not examined in the experiment over polysyllabic words, to confirm the statistical significance of their approximations, similar experiment is required. In addition, supported by the large amount of manual approximations, study of automatic approximation becomes possible. An algorithm is in proposing, in which, the key problems are to locate the turning points and to determine the pitch values of these points.

\section{ACKNOWLEDGEMENT}

This research is partially supported by two Earmarked Research Grants (Ref: CUHK 422704E and CUHK 413405) from the Hong Kong Research Grants Council.

\section{REFERENCES}

[1] P.B. Denes and E.N. Pinson, The Speech Chain: The Physics and Biology of Spoken Language, W.H. Freeman and Company, New York, 1998

[2] Y. Xu, "Understanding Tone from The Perspective of Production and Perception", Journal of Language and Linguistics, vol. 5(4), pp. 757-797, 2004

[3] Y. Xu, "Contextual Tonal Variations in Mandarin", Journal of Phonetics, vol. 25(1), pp. 61-83, 1997.

[4] Y.-J. Li, Tan Lee and Y. Qian, "Analysis and Modeling of F0 Contours for Cantonese Text-to-Speech", Journal of ACM Transactions on Asian Language Information Processing, vol. 3(3), pp. 169-180, 2004.

[5] J. Hart, R. Collier and A. Cohen, A Perceptual Study of Intonation: An Experimental-phonetic Approach to Speech Melody, Cambridge University Press, London, 1990.

[6] C. d'Alessandro and P. Mertens, "Automatic Pitch Contour Stylization Using A Model of Tonal Perception", Journal of Computer Speech and Language, vol. 9, pp. 257-288, 1995.

[7] X. Shen, M. Lin and J. Yan, "F0 Turning Point as An F0 Cue to Tonal Contrast: A Case Study of Mandarin Tones 2 and 3. Journal of Acoustical Society of America, vol. 93, pp. 2241-2243, 1993.

[8] E. Khouw and V. Ciocca, "Perceptual Correlates of Cantonese Tones", Journal of Phonetics, vol. 35, pp. 104-117, 2007.

[9] Linguistic Society of Hong Kong (LSHK), Hong Kong Jyut Ping Characters Table (粵語拼音字表). Linguistic Society of Hong Kong Press (香港語言學會出版), Hong Kong, 1997.

[10] Y.-J. Li and Tan Lee, "Perceptual Equivalence of Approximated Cantonese Tone Contours", Proceedings of Interspeech 2007, Belgium, pp. 2677-2680, 2007.

[11] W.K. Lo, Tan Lee and P.C. Ching, "Development of Cantonese Spoken Language Crpora for Speech Applications", Proceedings of ISCSLP, Singapore, pp. 102 - 107, 1998.

[12] P. Boersma and D. Weenink, Praat: Doing Phonetics by Computer (version 4.4.12.), http://www.fon.hum.uva.nl/praat, 2006. 